\title{
Role of integrons, plasmids and SXT elements in multidrug resistance of Vibrio cholerae and Providencia vermicola obtained from a clinical isolate of diarrhea
}

\section{Neha Rajpara ${ }^{1+}$, Braj M. R. N. S. Kutar ${ }^{1+}$, Ritam Sinha ${ }^{2}$, Dhrubajyoti Nag $^{2}$, Hemanta Koley ${ }^{2}$, Thandavarayan Ramamurthy ${ }^{2}$ and Ashima K. Bhardwaj ${ }^{1}$ *}

${ }^{1}$ Molecular Biology of Diseases, Department of Human Health and Diseases, School of Biological Sciences and Biotechnology, Indian Institute of Advanced Research, Gandhinagar, India

${ }^{2}$ Division of Bacteriology, National Institute of Cholera and Enteric Diseases, Kolkata, India

\section{Edited by:}

Tzi Bun Ng, The Chinese University of Hong Kong, China

\section{Reviewed by:}

M. Pilar Francino, Center for Public

Health Research, Spain

Amit Kumar, Kansas State University, USA

\section{*Correspondence:}

Ashima K. Bhardwaj, Molecular Biology of Diseases, Department of Human Health and Diseases, School of Biological Sciences and

Biotechnology, Indian Institute of Advanced Research, Koba Institutional Area, Gandhinagar 382 007, Gujarat, India

e-mail: ashima.bhardwaj@gmail.com

${ }^{t}$ These authors have contributed equally to this work.
The isolates of Vibrio cholerae and Providencia vermicola obtained from a diarrheal patient were investigated for genetic elements governing their drug resistance phenotypes. Out of 14 antibiotics tested, $V$. cholerae $V_{c}$ IDH02365 isolate showed resistance to nine antibiotics, while $P$. vermicola Pv NBA2365 was found to be resistant to all the antibiotics except polymyxin B. Though SXT integrase was depicted in both the bacteria, class 1 integron was found to be associated only with Pv NBA2365. Integrons in Pv NBA2365 conferred resistance to $\beta$-lactams, aminoglycosides, and trimethoprim. Pv NBA2365 carried two transformable plasmids imparting distinct antibiotic resistance traits to their Escherichia coli transformants. In rabbit ileal loop assays, Pv NBA2365 did not show any fluid accumulation (FA) in contrast with $V_{C}$ IDH02365 that showed high FA. To the best of our knowledge, this is the first report of a highly drug resistant $P$. vermicola and additionally co-existence of multidrug resistant $V$. cholerae and $P$. vermicola. Both the microbes appeared to possess a wide array of mobile genetic elements for a large spectrum of antimicrobial agents, some of which are being used in the treatment of acute diarrhea.

Keywords: Providencia vermicola, Vibrio cholerae, plasmid, integron, multidrug resistance, SXT element

\section{INTRODUCTION}

Diarrhea is one of the infectious diseases that pose a serious threat to humankind. It still remains a common cause of illness and death in developing countries and is caused by bacteria, viruses, or parasites (Navaneethan and Giannella, 2008). Cholera is one of these diarrheal diseases caused by the bacterium Vibrio cholerae. More than 200 serogroups of $V$. cholerae are known, out of which only two, O1 and O139 serogroups cause epidemics or outbreaks (Kaper etal., 1995; Faruque etal., 1998). Non-O1/non-O139 serogroups of $V$. cholerae have been associated with sporadic cases of diarrhea and have been implicated as progenitors of new variants of epidemic $V$. cholerae strains (Faruque et al., 2003). There are many reports of mixed infections of $V$. cholerae with other diarrheal pathogens such as Shigella spp., Salmonella spp., Campylobacter spp., and viruses (Faruque etal., 1994; Enzensberger et al., 2005; Mukherjee et al., 2009). Bacteria belonging to the genus Providencia are responsible for human infections such as urinary tract infections, endocarditis, ocular infections, traveler's diarrhea, and gastroenteritis (Murata et al., 2001; Krake and Tandon, 2004; Yoh et al., 2005; Koreishi et al., 2006). One of the members, Providencia vermicola, was first isolated from infective juveniles of the entomopathogenic nematode (Somvanshi et al., 2006). It has been shown to be a symbiont required for successful parasitic life of the nematode in the target insect such as oriental beetle (Young-Keun et al.,
2007). Due to its entomopathogenicity, the nematodes carrying P. vermicola are used as biopesticides (Young-Keun et al., 2007). The reports pertaining to the drug resistance of $P$. vermicola are non-existent.

Though the diarrheal diseases still remain a scourge, their treatment is becoming more complicated with the increasing problem of multiple drug resistance exhibited by the etiological agents (Sack et al., 2001; WHO, 2014). Emergence of multiple drug resistance causes a serious clinical problem like increase in morbidity and mortality rates (Dalsgaard et al., 2000; Ghosh and Ramamurthy, 2011). All the bacteria including Vibrio spp. and Providencia spp. have been reported to exhibit multidrug resistance which seriously hampers the containment of infections caused by them (Marchandin et al., 1999; Ghosh and Ramamurthy, 2011). There are several different mechanisms by which bacteria are known to acquire/disseminate drug resistance. Some of the mechanisms are mobile genetic elements such as integrons, transposons and plasmids and numerous inherent mechanisms like mutations in target genes or efflux pumps, to name a few (Ghosh and Ramamurthy, 2011; Bhardwaj and Mohanty, 2012; Bhardwaj et al., 2014). Mobile genetic elements play a crucial role in the rapid acquisition and dissemination of drug resistance genes thus manipulating the genome plasticity in accordance with the changing environment (Bhardwaj et al., 2014). Integrons work as platforms that acquire open reading frames by site-specific recombination and convert them into 
functional form by their expression driven by a resident promoter at the $5^{\prime}$ end of integrons (Stokes and Hall, 1989). SXT element is an integrative and conjugative element (ICE) that acts as a vehicle for translocating many genes including the antibiotic resistance genes (Waldor et al., 1996). This element was first described in $V$. cholerae MO10 isolated from Madras, India in 1992, where it encoded resistance to trimethoprim, sulfamethoxazole, streptomycin, and chloramphenicol (Waldor et al., 1996). ICEs are a type of conjugative transposons that are not capable of autonomous replication and therefore, they integrate and replicate with the chromosomal DNA of the host bacterium (Burrus and Waldor, 2004). Plasmids known as $\mathrm{R}$ plasmids ( $\mathrm{R}$ for resistance), capable of autonomous replication, play an important role in the spread of antibiotic resistance among bacteria (Sack et al., 2001). Earlier work from this laboratory has described the role of some of these genetic elements in determining the multidrug resistance phenotype displayed by the clinical isolates of $V$. cholerae and Vibrio fluvialis from Kolkata, India (Rajpara et al., 2009; Mohanty et al., 2012; Singh et al., 2012; Kutar et al., 2013). In addition, inhibition of efflux pumps and quorum sensing as alternatives for control of multidrug resistance have also been reviewed (Bhardwaj and Mohanty, 2012; Bhardwaj et al., 2013). Present study describes an interesting case of mixed occurrence of $V$. cholerae and P. vermicola in a stab culture from a clinical sample of a diarrheal patient. Results reveal the exquisite contribution/interplay of plasmids, integrons, and SXT elements in imparting the multidrug resistance traits to both the organisms.

\section{MATERIALS AND METHODS \\ BACTERIAL ISOLATES, THEIR IDENTIFICATION, AND DNA PREPARATION} Vibrio cholerae (referred to as Vc IDH02365) and Providencia vermicola (referred to as Pv NBA2365) were isolated from a stab culture. This stab culture was obtained from the alkaline peptone water (Atlas, 2010) enriched diarrheal sample from a 37 years old male patient with acute watery diarrhea and severe dehydration admitted to the Infectious Diseases Hospital (IDH), Kolkata, India, in 2009. The procedure of obtaining patient sample and his consent was approved by the Institutional Ethical Clearance Committee, National Institute of Cholera and Enteric Diseases (NICED), Kolkata, which conducts an active surveillance of diarrheal diseases at the IDH, Kolkata. All the strains used in the study are described in Table 1. For identification of the bacterial isolates, biochemical analysis was performed by the standard protocols (WHO, 1987). 16S rRNA sequencing was performed to confirm the identity of $P$. vermicola using the primers $8 \mathrm{~F}$ and $1492 \mathrm{R}$ (Table 1). Genomic and plasmid DNA extraction was performed as described previously (Thungapathra et al., 2002). The study was approved by the Institutional Biosafety Committee (IBSC) of the Indian Institute of Advanced Research, Gandhinagar, and Review Committee on Genetic Manipulation (RCGM) governed by guidelines laid down by the Department of Biotechnology, Government of India.

\section{PULSED FIELD GEL ELECTROPHORESIS (PFGE) AND RANDOM AMPLIFICATION OF POLYMORPHIC DNA (RAPD) ANALYSIS}

Pulsed field gel electrophoresis (PFGE) analysis was carried out as described previously (Parsons et al., 2007). For PFGE, 1\%
Bio-Rad pulsed field certified agarose gel (Bio-Rad Laboratories, Richmond, CA, USA) was prepared in 0.5X TBE and run in CHEF MAPPER (Bio-Rad Laboratories) using autoalgorithm mode (molecular weight range: $100-350 \mathrm{~K}$ and a run time of $19 \mathrm{~h}$ ). The gel was stained with $0.05 \mathrm{mg} \mathrm{ml}^{-1}$ ethidium bromide for $30 \mathrm{~min}$ and destained with sterile water for $1 \mathrm{~h}$. RAPD analysis was carried out by 1281 and 1283 primers as described earlier (Akopyanz et al., 1992; Chakraborty et al., 2001).

\section{ANTIMICROBIAL SUSCEPTIBILITY TESTS}

The isolates were tested for their susceptibility to ampicillin (10 $\mu \mathrm{g})$, chloramphenicol (30 $\mu \mathrm{g})$, co-trimoxazole $(1.25 \mu \mathrm{g}$ trimethoprim/23.75 $\mu \mathrm{g}$ sulfamethoxazole), ciprofloxacin $(5 \mu \mathrm{g})$, gentamicin $(10 \mu \mathrm{g})$, streptomycin $(10 \mu \mathrm{g})$, sulfisoxazole $(300 \mu \mathrm{g})$, trimethoprim $(5 \mu \mathrm{g})$, tetracycline $(30 \mu \mathrm{g})$, neomycin $(30 \mu \mathrm{g})$, nalidixic acid $(30 \mu \mathrm{g})$, norfloxacin $(10 \mu \mathrm{g})$, kanamycin $(30 \mu \mathrm{g})$, and polymyxin B (300 units) by the disk diffusion method using commercial disks (HiMedia, Mumbai, India) in accordance with the criteria recommended by Clinical and Laboratory Standards Institute (CLSI) standards (CLSI, 2010). When no interpretive criteria for $V$. cholerae were available based on CLSI guidelines, breakpoints for Enterobacteriaceae were applied (CLSI, 2010). For $P$. vermicola, breakpoints for Enterobacteriaceae were applied as it belongs to the same family. Escherichia coli ATCC 25922 was used for quality control. Experiments were performed at least two times.

\section{POLYMERASE CHAIN REACTIONS (PCRs)}

All the primers used in this study have been described in Table 1. Polymerase chain reaction (PCR) for analysis of SXT elements and class 1 integrons were carried out as described before (Rajpara et al., 2009). Primer pairs L2/L3 (specific for $5^{\prime}$ conserved segment [ $5^{\prime} \mathrm{CS}$ ] of class 1 integron), qacE $\Delta 1 /$ Sul1B (specific for $3^{\prime}$ conserved segment [ $3^{\prime} \mathrm{CS}$ ] of class 1 integron), In-F/In-B (specific for variable region of class 1 integron) were used for detection and characterization of class 1 integrons using the same conditions excepting that for the primer pair In-F/In-B, annealing was carried out at $63.5^{\circ} \mathrm{C}$ for $1 \mathrm{~min}$. Reactions were also performed for confirmation of $V$. cholerae isolate using OmpW-specific primer, and confirmation of $P$. vermicola isolate using the species-specific primers Provi_F, Provi_R, and P_Vermi_R. In PCR for Providencia sp. (using Provi_F and Provi_R to detect both $P$. rettgeri and P. vermicola), annealing was carried out at $62^{\circ} \mathrm{C}$ for $1 \mathrm{~min}$ and polymerization was carried out at $72^{\circ} \mathrm{C}$ for $1.5 \mathrm{~min}$, while in PCR for P. vermicola (using Provi_F and P_Vermi_R to detect only $P$. vermicola and not $P$. rettgeri), annealing was carried out at $68^{\circ} \mathrm{C}$ for $1 \mathrm{~min}$ and polymerization was carried out at $72^{\circ} \mathrm{C}$ for $1.5 \mathrm{~min}$. PCR reactions were performed using a PTC-225 DNA Engine Tetrad ${ }^{\mathrm{TM}}$ Cycler (MJ Research Inc., Waltham, MA, USA). Recombinant Taq DNA polymerase (Fermentas International Inc., Burlington, ON, Canada) was used along with appropriate buffers.

\section{TRANSFORMATION}

Transformation of E. coli JM109 was carried out by electroporation (Gene Pulser XCell; Bio-Rad Laboratories, Richmond, CA, USA) using $200 \mathrm{ng}$ of total DNA or plasmid DNA from Pv NBA2365 and Vc IDH02365 isolates. Transformants were selected on 
Table 1 | List of strains and primers used in the study.

\begin{tabular}{|c|c|c|}
\hline \multicolumn{3}{|c|}{ Strains } \\
\hline Name of the strain & Description & Reference \\
\hline E. coli JM109 & Host for electroporation experiments & Yanisch-Perron et al. (1985) \\
\hline Vibrio fluvialis BD146 & Positive control for class 1 integron & Rajpara et al. (2009) \\
\hline V. cholerae O1 El Tor N16961 & Control for RAPD analysis & Heidelberg et al. (2000) \\
\hline V. cholerae 01 classical 569B & Reference strain in antibiogram analysis & Kennedy and Richardson (1969) \\
\hline V. cholerae O1 El Tor IDH01526 & Control for V. cholerae-specific protein OmpW PCR & Kutar etal. (2013) \\
\hline V. cholerae O1 El Tor IDH01572 & control for PFGE experiments & Kutar et al. (2013) \\
\hline V. cholerae O1 El Tor IDH01581 & Control for PFGE experiments & Kutar et al. (2013) \\
\hline & & Biotechnology Mission, Gujarat, India \\
\hline Providencia rettgeri MTCC8099 & Control for Providencia-specific PCR & Microbial type culture collection, Chandigarh, India \\
\hline \multicolumn{3}{|c|}{ Primers } \\
\hline Name of primer & Primer sequence $\left(5^{\prime}->3^{\prime}\right)$ & Reference \\
\hline $8 \mathrm{~F}$ & AGAGTTTGATCCTGGCTCAG & Eden etal. (1991) \\
\hline $1492 R$ & ACGGCTACCTTGTTACGACTT & Eden etal. (1991) \\
\hline Provi_F & CGCATAATCTCTTAGGAGCAAA & This study \\
\hline L2 & GACGATGCGTGGAGACC & Maguire et al. (2001) \\
\hline L3 & CTTGCTGCTTGGATGCC & Maguire et al. (2001) \\
\hline $\ln F$ & GGCATCCAAGCAGCAAGC & Dalsgaard et al. (2000) \\
\hline $\ln B$ & AAGCAGACTTGACCTGAT & Dalsgaard et al. (2000) \\
\hline Qac E $\Delta 1-\mathrm{F}$ & ATCGCAATAGTTGGCGAAGT & Dalsgaard et al. (2000) \\
\hline Sul1B & GCAAGGCGGAAACCCGCC & Dalsgaard et al. (2000) \\
\hline SXT-F & TTATCGTTTCGATGGC & Thungapathra et al. (2002) \\
\hline SXT-R & GCTCTTCTTGTCCGTTC & Thungapathra et al. (2002) \\
\hline
\end{tabular}

Luria-Bertani (LB; Lennox, 1955; Atlas, 2010) plates containing ampicillin $\left(25 \mu \mathrm{g} \mathrm{ml}^{-1}\right)$. Electrocompetent cell preparation and electroporation preset protocols used for E. coli cells were as per the manufacturer's instructions.

\section{DNA SEOUENCING AND SEOUENCE ANALYSIS}

DNA segments amplified from integrons and SXT integrases were sequenced. DNA sequencing was performed by Sanger's chain termination method using DNA sequencer (Applied Biosystems; 3730/3730xl DNA analyzer). For larger segments of DNA, primer walking was carried out and the sequence was assembled. The assembled sequences were analyzed by BLAST search and the sequences were submitted to GenBank. The ORF (Open Reading Frame) Finder tool at the National Center for Biotechnology Information (NCBI) website ${ }^{1}$ was used to predict all the possible ORFs in these sequences.

\footnotetext{
${ }^{1}$ http://www.ncbi.nlm.nih.gov/gorf/gorf.html
} 


\section{RABBIT ILEAL LOOP ASSAY}

Rabbit ileal loop experiments were conducted as described earlier (De and Chatterje, 1953; Rajpara et al., 2013). New Zealand White male rabbits $(1.5-2 \mathrm{~kg})$ were fasted for $48 \mathrm{~h}$ prior to surgery and fed only water ad libitum. Rabbits were anesthetized by intramuscular administration of ketamine ( $35 \mathrm{mg} \mathrm{kg}^{-1}$ of body weight) and xylazine $\left(5 \mathrm{mg} \mathrm{kg}^{-1}\right)$. A laparotomy was performed, and the ileum was washed and ligated into discrete loops of approximately $10 \mathrm{~cm}$. Each loop was inoculated with $10^{8} \mathrm{CFU}$ of challenge strain (V. cholerae O1 El Tor N16961, Vc IDH02365, Pv NBA2365 and the mixture of Vc IDH02365 and Pv NBA2365) in phosphate-buffered saline (PBS). PBS was used as negative control. The intestine was returned to the peritoneum and the animals were sutured and returned to their cages. After $18 \mathrm{~h}$, rabbits were sacrificed by intravenous injection of pentobarbital $\left(150 \mathrm{mg} \mathrm{kg}^{-1}\right)$, and the loops were excised. Fluid volume and loop length were measured, and secretion was recorded as milliliters per centimeter. For rabbit ileal loop assay, the study was approved by the Institutional Ethical Clearance Committee of NICED. All surgery was performed under sodium pentobarbital anesthesia, and all efforts were made to minimize suffering.

\section{GenBank SUBMISSIONS}

The accession numbers for the gene sequences submitted to GenBank from this study were: KC709647, KC709648 for sequences of SXT integrases from Vc IDH02365 and Pv NBA2365 respectively, KC709650, KC709653, KC709649, KC709652 for 5' CS, 3' $\mathrm{CS}, 1.2 \mathrm{~kb}$ variable region and $2.8 \mathrm{~kb}$ variable region respectively of class 1 integron from Pv NBA2365, and KC709651 for 16SrRNA of Pv NBA2365.

\section{RESULTS}

\section{CLINICAL ISOLATE CONSISTED OF V. cholerae AND P. vermicola}

The clinical isolate from diarrhea patient was received in the form of a stab culture from NICED as part of a collection of $119 \mathrm{~V}$. cholerae isolates of 2009. It showed the growth of two bacteria with different colony morphologies, i.e., one yielded big sized (4 $\mathrm{mm}$ in diameter) mucoid yellowish colonies and the other bacterium yielded small ( $2 \mathrm{~mm}$ in diameter) off white colonies on LB medium. On thiosulfate-citrate-bile salts-sucrose (TCBS) agar (Morris et al., 1979; MacFaddin, 2000), V. cholerae grew as sucrose fermenting yellow color colonies, while the other bacteria appeared as bluish green colonies. These bluish green colonies turned yellow when incubated for durations longer than $24 \mathrm{~h}$. Genomic DNA was isolated from both the sucrose fermenting and non-fermenting colonies and analyzed in $V$. cholerae-specific OmpW PCR. An amplicon of expected size (586 bp) was obtained only with DNA from sucrose fermenting colonies confirming its identity as $V$. cholerae whereas the DNA from sucrose-nonfermenting colonies did not yield this amplicon (Figure 1A).
A

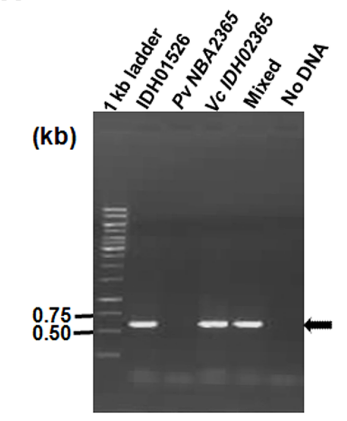

C

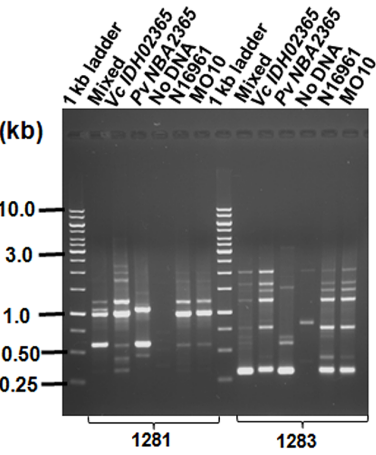

FIGURE 1 | Identification and differentiation of constituent bacteria of a clinical isolate of diarrhea. Agarose gel (1\%) analysis of (A) PCR products for confirming $V$. cholerae species with primer pair OmpWF/OmpW-R; (B) PFGE of DNA from Vc IDH02365 and P. vermicola PV NBA2365 digested with Sfil restriction enzyme; (C) RAPD profile of clinical isolates with 1281 and 1283 primers; and (D) PCR with species-specific primers for $P$. rettgeri/P. vermicola and only $P$. vermicola.

B

D

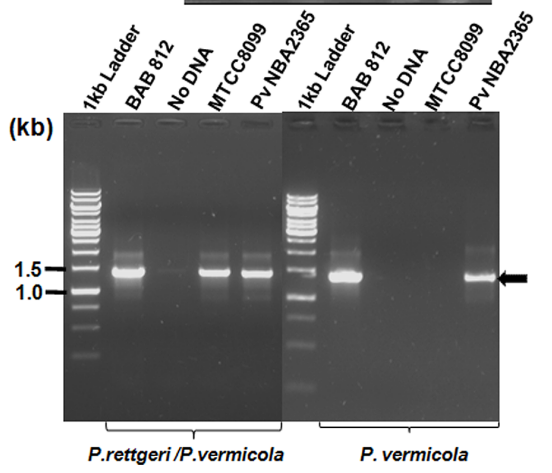

All the DNA samples used as templates are indicated on top of each lane. The clinical isolates of $V$. cholerae IDH01526, IDH01572, IDH01581, N16961, MO10, P. vermicola BAB 812, P. rettgeri MTCC8099 and mixture of $V_{c}$ IDH02365 and Pv NBA2365 as obtained from the original stab culture (mixed) were used as controls for various assays. Marker positions in $\mathrm{kb}$ have been indicated in the left of each panel. Arrows indicate the position of relevant amplicons for each PCR. 
Table 2 | Biochemical tests and morphological analysis of Vc IDH02365 and Pv NBA2365.

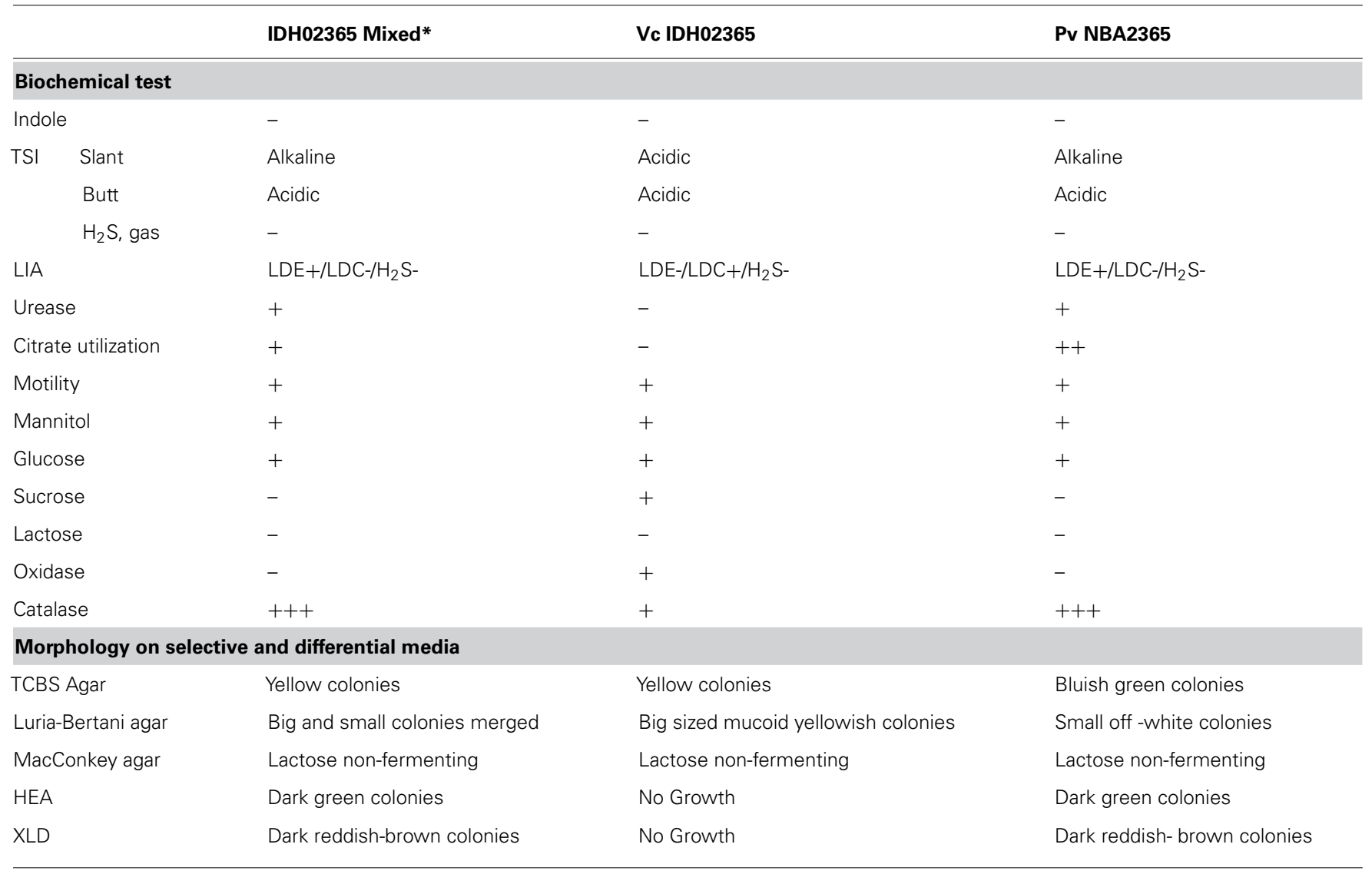

Phenotypes recorded after $18-20 \mathrm{~h}$ at $37^{\circ} \mathrm{C}$.

TSI, triple sugar iron agar; LIA, lysine iron agar; TCBS, thiosulfate citrate bile salt sucrose; HEA, Hektoen enteric agar; XLD, xylose lysine desoxycholate agar; LDE, lysine deaminase; LDC, lysine decarboxylase.

${ }^{*}$ Clinical isolate from diarrheal patient consisting of mixture of two bacteria (VC IDH02365 and PV NBA2365) as obtained from the stab culture.

Table 3 | Antibiotic susceptibility profiles of Pv NBA2365, Vc IDH02365, and other reference strains.

\begin{tabular}{|c|c|c|c|c|c|c|}
\hline & Pv NBA2365 & $\begin{array}{l}\text { P. vermicola } \\
\text { BAB812 }\end{array}$ & $\begin{array}{l}\text { P. vermicola } \\
\text { BAB813 }\end{array}$ & Vc IDH02365 & $\begin{array}{l}\text { V. cholerae } 01 \\
\text { EI Tor N16961 }\end{array}$ & $\begin{array}{l}\text { V. cholerae } \\
569 \mathrm{~B}\end{array}$ \\
\hline
\end{tabular}

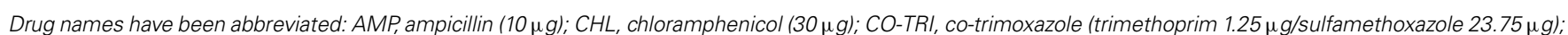

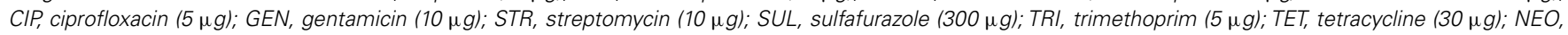

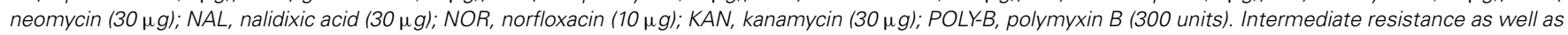
complete resistance were considered to be a resistance trait.

Distinct band patterns obtained by PFGE and RAPD analysis in these two colonies also corroborated the presence of two different bacteria (Figures 1B,C). Both the colonies were subjected to a variety of biochemical tests (Table 2 ). The organism constituting the small sucrose non-fermenting colony was found to be oxidasenegative as compared to $V$. cholerae which was oxidase-positive. Therefore, as evident from many other biochemical tests and growth patterns on various media, this clinical isolate consisted of $V$. cholerae and another organism with biochemical and morphological phenotype different from $V$. cholerae (Table 2). In order to establish the identity of the non-vibrio bacteria, results of their biochemical tests were fed into ABIS online (Advanced Bacterial Identification Software ${ }^{2}$ ). Analysis indicated the possibility of this bacterium to be Providencia rettgeri or P. vermicola. $16 \mathrm{~S}$ rRNA

\footnotetext{
${ }^{2}$ http://www.tgw1916.net/bacteria_logare.html
} 
sequencing was carried out and the sequence established that this bacterium was P. vermicola (GenBank submission KC709651). Further confirmation using PCR assay with the primers specific for both the species (yielding a major band of expected size in $P$. vermicola samples BAB812, Pv NBA2365 as well as $P$. rettgeri sample MTCC8099) or primers specific only for $P$. vermicola (yielding a prominent band only in P. vermicola samples BAB812 and Pv NBA2365) clearly proved the bacterium to be $P$. vermicola (Figure 1D). Hereafter, these bacteria were termed as Vc IDH02365 (for this V. cholerae) and Pv NBA2365 (for this $P$. vermicola).

\section{Vc IDH02365 AND Pv NBA2365 SHOWED DISTINCT ANTIBIOTIC RESISTANCE PROFILES}

Vc IDH02365 was resistant to co-trimoxazole, nalidixic acid, polymyxin-B, sulfisoxazole and trimethoprim and showed intermediate resistance to ampicillin, ciprofloxacin, streptomycin, and tetracycline. $\mathrm{Pv}$ NBA2365 showed complete/intermediate resistance to all the antibiotics except polymyxin-B (Table 3). The resistance of Pv NBA2365 to all the antibiotics used in the study was noteworthy. For comparison, other strains of $P$. vermicola and $V$. cholerae were tested in this study. The results showed distinct resistance profiles for various strains of both the organisms (Table 3).

\section{PRESENCE OF SXT ELEMENT IN Vc IDH02365 AND Pv NBA2365}

As the antibiotic resistance profiles of both the bacteria were characteristic of SXT element, PCR experiment was carried out to analyze the presence of this element in these isolates by amplification of SXT integrase. The experiment revealed that both the isolates were positive for SXT integrase (Figure 2A) since $1.0 \mathrm{~kb}$ amplicon was obtained in Vc IDH02365 as well as PV NBA2365. Sequences of these amplicons were analyzed by BLAST search at NCBI site. Results showed that SXT integrase from Vc IDH02365 (GenBank submission KC709647) had 99\% identity with a large number of integrase sequences including the ones from the strains ICEVflInd 1 from an Indian isolate of $V$. fluvialis (GQ463144), ICEVchInd5 from Sevagram, India (GQ463142), ICEVchBan5 from Bangladesh ( $\underline{\text { GQ463140 }}$ ), and VC1786ICE sequence from Haiti outbreak ( $\underline{\mathbf{N 6 4 8 3 7 9}}$ ). SXT integrase from Pv NBA2365 (GenBank submission KC709648) had 98\% similarity with integrases from ICEPdaSpal from Photobacterium

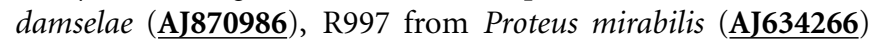
and ICE $V$ chInd4 from $V$. cholerae (GQ463141). There was 95\% similarity between these two SXT integrase nucleotide sequences from Vc IDH02365 and Pv NBA2365.

\section{Pv NBA2365 CONTAINED TWO CLASS 1 INTEGRONS}

To determine the presence of class 1 integrons as carriers of drug resistance genes, PCR reactions were performed with the primers specific for class 1 integron as described in methods. PCR with primers L2/L3 specific for $5^{\prime}$ CS of class 1 integron yielded an amplicon of around $300 \mathrm{bp}$ in Pv NBA2365 of which 234 bp were sequenced (GenBank submission KC709650; Figure 2B). Similarly, 3' CS of about 800 bp (GenBank submission KC709653) was obtained with qacE $\Delta 1 / \mathrm{Sul1B}$ primer pair in the same organism (Figure 2C). These amplicons were not obtained in Vc

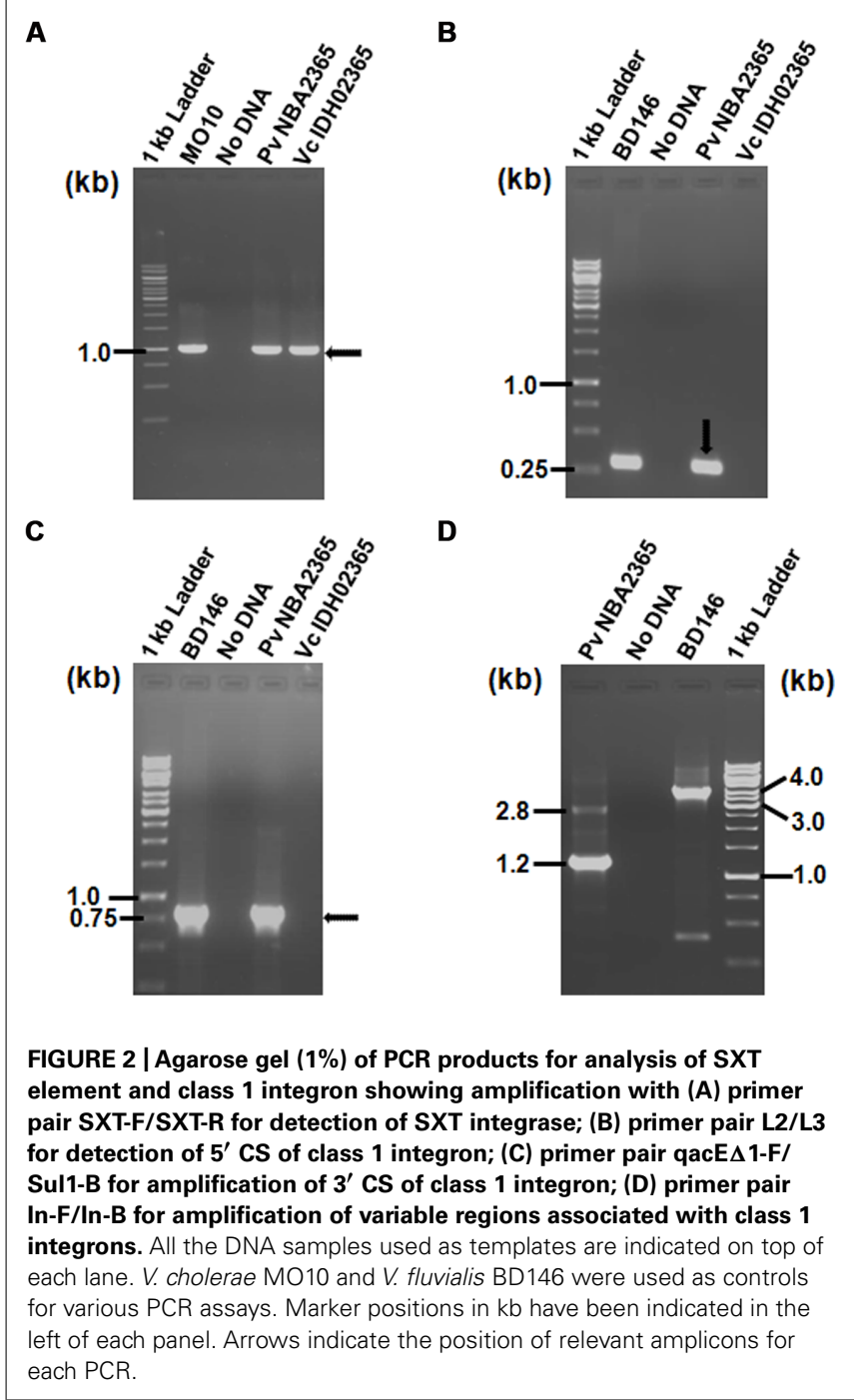

IDH02365. Moreover, the integron was associated with plas$\operatorname{mid}(\mathrm{s})$ in $\mathrm{Pv}$ NBA2365 as the bands corresponding to $5^{\prime} \mathrm{CS}$ and $3^{\prime}$ CS were obtained with plasmid DNA preparation. Analysis of variable region of integrons amplified using In-F/In-B primers indicated the presence of two major amplicons of 1.2 and $2.8 \mathrm{~kb}$ size (Figure 2D). The $1.2 \mathrm{~kb}$ amplicon (GenBank submission KC709649) contained gene cassettes dfrA1 (encoding group A drug-insensitive dihydrofolate reductase, which is responsible for trimethoprim resistance) and orfC (encoding a hypothetical protein). Amplicon with $2.8 \mathrm{~kb}$ length (GenBank submission KC709652) contained the cassettes blaVIM-1, aadB, $d f r A 1$, and orfC encoding metallo- $\beta$-lactamase, aminoglycoside2 "-adenyl transferase, group A drug-insensitive dihydrofolate reductase and hypothetical protein respectively (Figure 3). Therefore, these two integrons present in Pv NBA2365 mediated resistance to ampicillin, trimethoprim, gentamicin, tobramycin, and kanamycin. As was evident from the sequences and the results described above, 1.2 and $2.8 \mathrm{~kb}$ cassettes shared some common genes and the identity of $99 \%$ in those gene segments. 

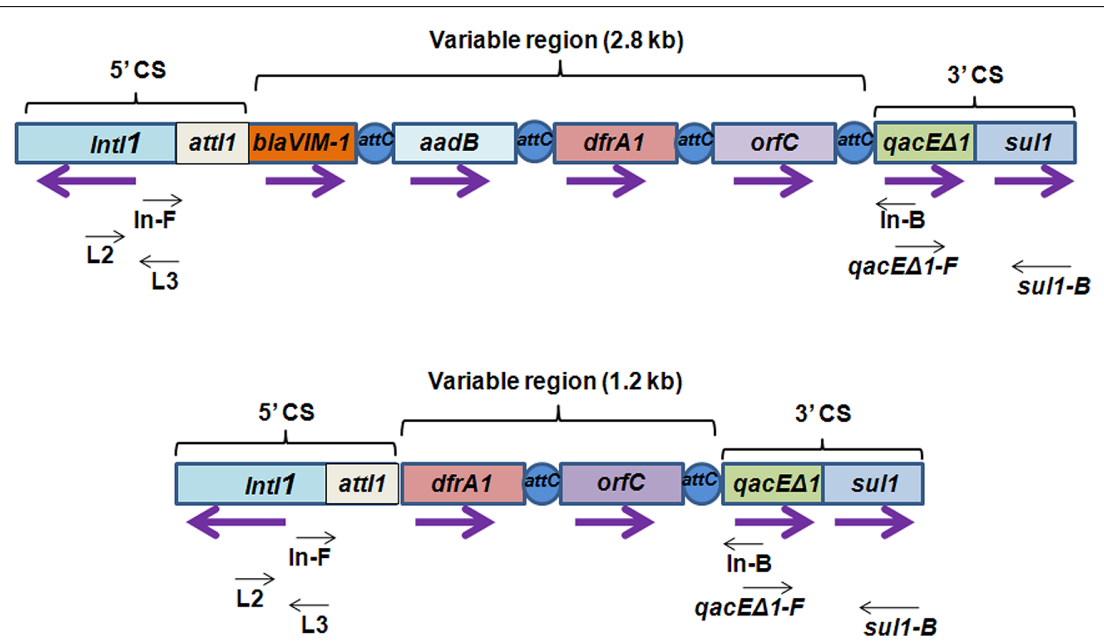

FIGURE 3 | Schematic representation of class 1 integron containing 2.8 and $1.2 \mathrm{~kb}$ variable regions harboring different kinds of gene cassettes. Integrons consist of a gene int/1 encoding a site-specific recombinase called "integrase" belonging to tyrosine-recombinase family and a recombination site att/1 into which exogenous gene cassettes (here blaVIM-1, aadB, dfrA1, and $\operatorname{orfC}$ ) harboring the recombination site att $C$ are inserted through site-specific recombination. These exogenous cassettes that vary from one integron to the other, together constitute variable region of the integron. In the $5^{\prime}$ conserved sequences ( $5^{\prime} \mathrm{CS}$ ), a promoter located within int $/ 1$ drives transcription of the captured genes. qacE 1 and sul1 are conserved regions in $3^{\prime}$ conserved sequences ( $3^{\prime} \mathrm{CS}$ ) which contribute resistance to ethidium bromide and sulfonamides. Purple arrows indicate the direction of transcription for each gene. Black arrows indicate the position of primers used for detection and analysis of class 1 integrons.

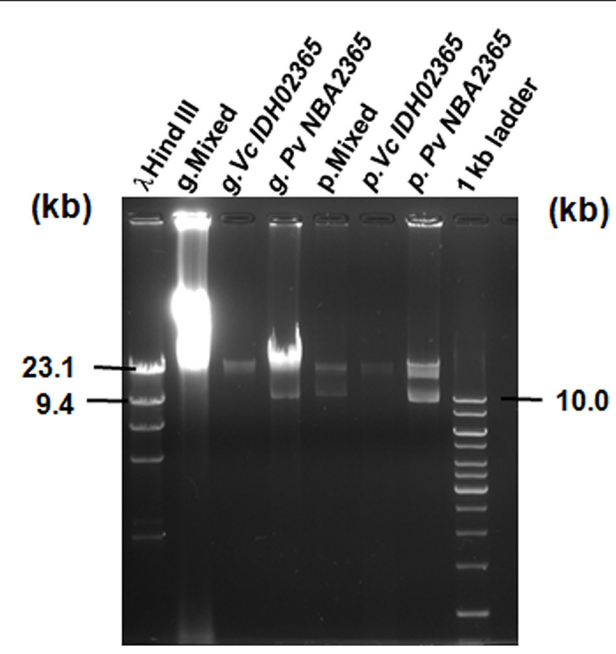

FIGURE 4 | Agarose gel analysis for genomic DNA and plasmid DNA of Vc IDH02365 and Pv NBA2365. All the DNA samples used as templates are indicated on top of each lane. Marker positions in kb have been indicated. g. denotes total genomic DNA preparation and p. denotes plasmid DNA preparation.

\section{PRESENCE OF PLASMIDS IN Pv NBA2365 AND Vc IDH02365}

Agarose gel analysis of total genomic and plasmid DNA from $\mathrm{PV}$ NBA2365 and Vc IDH02365 indicated the presence of multiple plasmid bands in the range of 9.0-23.0 kb (Figure 4). To determine the transferability of these plasmids, transformation experiments were carried out and ampicillin selection was used. Conjugation could not be performed as PvNBA2365 was resistant to all the drugs that were tested and therefore, screening of the exconjugants was a problem. Transformants were obtained with the
DNA preparations from these bacterial isolates confirming the presence of transferable plasmids in both the bacteria. These transformants were analyzed by agarose gel electrophoresis of their plasmid DNA, antibiotic susceptibility profiles and for integron analysis. Two types of transformants were obtained. First type was negative for integrase of class 1 integron and showed resistance to ampicillin, kanamycin, nalidixic acid, and neomycin (Table 4). These transformants were obtained with DNA from both the bacteria. The second type of transformants positive for this integrase, were obtained only with DNA from Pv NBA2365 and displayed resistance to ampicillin, co-trimoxazole, nalidixic acid, kanamycin, sulfisoxazole, and trimethoprim (Table 4). This indicated that there were atleast two plasmids associated with $\mathrm{Pv}$ NBA2365.

\section{Vc IDH02365 WAS HIGHLY ENTEROTOXIC IN A RABBIT ILEAL LOOP ASSAY AS COMPARED TO Pv NBA2365}

Vc IDH02365 and Pv NBA2365 were tested in rabbit ileal loop assays to assess their fluid accumulation (FA) potential (Figure 5). PBS, the mixture of Vc IDH02365 and Pv NBA2365, and $V$. cholerae O1 El Tor N16961 were taken as controls in this assay. Results revealed that the FA was the highest for $\mathrm{Vc}$ IDH02365 $\left(2.7 \mathrm{ml} \mathrm{cm}^{-1}\right)$ which was comparable to that of the epidemic strain $V$. cholerae O1 El Tor N16961 $\left(2.1 \mathrm{ml} \mathrm{cm}^{-1}\right)$. Pv NBA2365 showed the accumulation of $0.07 \mathrm{ml} \mathrm{cm}^{-1}$. The controls of PBS and the mixture of isolates of Pv NBA2365 and Vc IDH02365 showed the FA values of 0.02 and $0.08 \mathrm{ml} \mathrm{cm}^{-1}$ respectively.

\section{DISCUSSION}

Bacteria show remarkable adaptability for their survival under stressful conditions (Lee et al., 2010). They evolve rapidly not only 
Table 4 | Antibiotic susceptibility patterns of Vc IDH02365, Pv NBA2365 and their respective E. coli JM109 transformants.

\begin{tabular}{lll}
\hline Strains & Vc IDH02365 & Pv NBA2365 \\
\hline Parent & CO-TRI, NAL, POLY-B, SUL, TRI, & AMP, CIP, CO-TRI, GEN, NAL, NOR, SUL, \\
& AMP, CIP, STR, TET & TET, TRI, KAN, CHL, NEO, STR \\
Integrase-positive transformants & NO TRANSFORMANTS & AMP, CO-TRI, NAL, SUL, TRI, KAN \\
Integrase-negative transformants & AMP, KAN, NAL, NEO & AMP, KAN, NAL, NEO
\end{tabular}

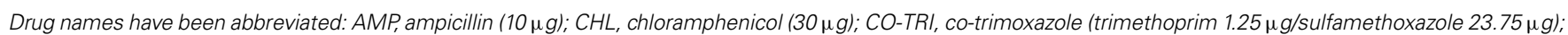
CIP, ciprofloxacin $(5 \mu \mathrm{g})$; GEN, gentamicin $(10 \mu \mathrm{g})$; STR, streptomycin $(10 \mu \mathrm{g})$; SUL, sulfafurazole $(300 \mu \mathrm{g})$; TRI, trimethoprim $(5 \mu \mathrm{g})$; TET, tetracycline $(30 \mu \mathrm{g})$; NEO, neomycin $(30 \mu \mathrm{g}) ; \mathrm{NAL}$, nalidixic acid $(30 \mu \mathrm{g})$; NOR, norfloxacin $(10 \mu \mathrm{g}) ; \mathrm{KAN}$, kanamycin $(30 \mu \mathrm{g}) ;$ POLY-B, polymyxin B $(300$ units). Two transformants from each kind of DNA sample were tested for their antibiograms. The experiment was done in duplicates. Intermediate resistance as well as complete resistance were considered to be a resistance trait. E. coli JM109 was resistant to nalidixic acid.

A

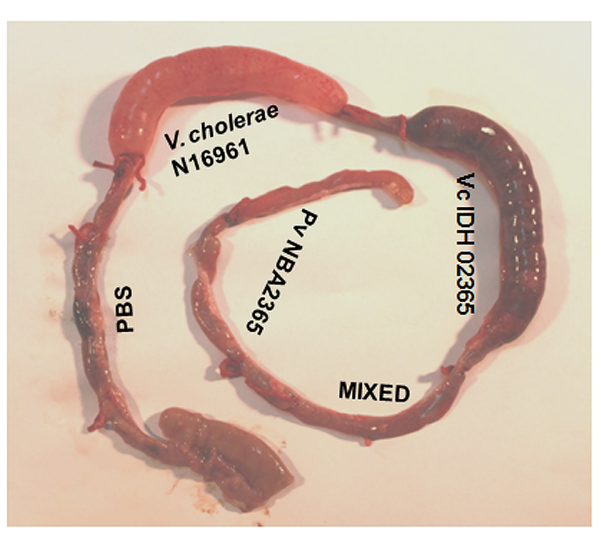

FIGURE 5 | Rabbit ileal loop assay to assess entrotoxigenic activity. Pictorial view of rabbit ileal loop of different clinical strains (A). Analysis of fluid accumulation of different clinical strains (B). Rabbit ileal loops were inoculated with $10^{8} \mathrm{CFU}$ of each strain V. cholerae N16961 (O1 El Tor, clinical),
B

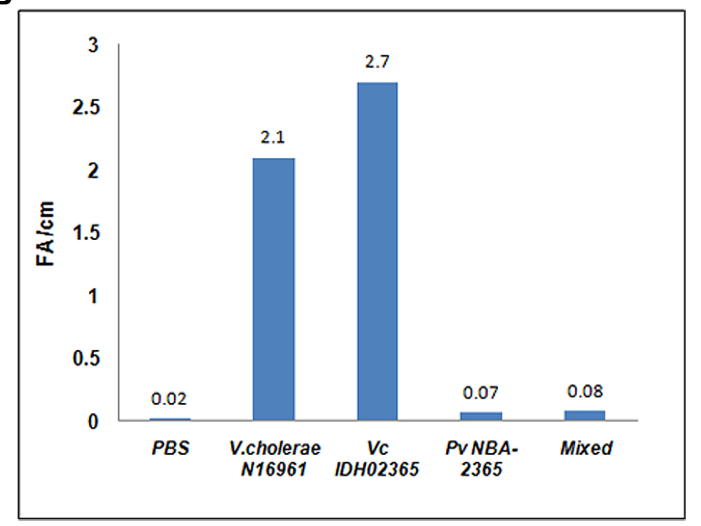

Vc IDH02365 (O1 El Tor, clinical), Pv NBA2365 in PBS and incubated for $18 \mathrm{~h}$. Results are expressed as fluid accumulation (FA; in milliliters) per loop length (in centimeters). PBS and the mixture of Pv NBA2365 and Vc IDH02365 were taken as controls in this assay. by mutations and rapid multiplication, but also by transfer of DNA, which can result in strains with beneficial mutations from more than one parent. Another way is communication/quorum sensing by autoinducers for their mutual survival and fitness (Macleod and Stickler, 2007; Lee et al., 2010). In addition, mobile genetic elements play crucial role in adaptation and evolution through horizontal gene transfer which involves panoply of drug resistance genes and many other genes required to adapt to newer situations (Bhardwaj and Vinothkumar, 2015). In this study, results have been presented for a diarrheal sample containing $V$. cholerae Vc IDH02365 and P. vermicola Pv NBA2365. Presence of Pv NBA2365 with Vc IDH02365 could actually be derived from the clinical sample of diarrhea itself (co-infection) or could have been derived as a contamination of the stab culture (contaminant). The possible contamination of the stab could be ruled out as no other strain in the population of 119 strains of $V$. cholerae of 2009 (from where Vc IDH02365 was obtained) harbored P. vermicola.

From the data obtained, it appeared that both the organisms utilized a diverse array of genetic elements to manage their survival under pressure from various classes of antibiotics. The high degree of drug resistance harbored by Pv NBA2365 could be of special concern as the organism was found equipped with all possible mobile genetic elements; multiple plasmids, multiple integrons, and SXT element. This clearly reflects the rapid evolution of these organisms through acquisition and dissemination of antibiotic resistance traits in the environment.

Analysis of the antibiotic susceptibility patterns revealed that Vc IDH02365 was resistant to nine and Pv NBA2365 was resistant to thirteen out of the 14 antibiotics used in the study. In order to unravel the molecular mechanisms responsible for the acquisition/dissemination of their drug resistance phenotypes, a study was initiated to understand the contribution of various mobile genetic elements such as SXT element, integrons, and plasmids. Analysis of integrase for SXT element revealed that this element was present in both the organisms. These integrases were 95\% identical at nucleotide level and 99\% identical at amino acid level. $P$. rettgeri from South Africa was reported to carry an SXT-related IncJ element called R391 in 1967 (Coetzee et al., 1972) and it was hypothesized that Providencia spp. could be a primary source of SXT element (Burrus et al., 2006). SXT and R391 integrase genes have been shown to be nearly identical (Hochhut etal., 2001). Comparison of the complete genome sequences of SXT and R391 has also proved the close relationship 
between these two ICE elements (Beaber et al., 2002). Therefore, though in this study, the complete sequences were not determined for SXT elements from Pv NBA2365 and Vc IDH02365, our findings from the nearly identical nucleotide sequences of SXT integrase genes from these organisms corroborated the earlier findings made by other groups (Hochhut et al., 2001; Beaber et al., 2002).

Integrons were only harbored by Pv NBA2365 and conferred resistance to ampicillin, trimethoprim, gentamicin, tobramycin, and kanamycin. The $1.2 \mathrm{~kb}$ variable region of class 1 integron was $99 \%$ identical (100\% query coverage) to the inetgron sequences from many Salmonella enterica sp. (Y963803.6, EF547513.1, DQ641477.1), $V$. cholerae non-O1/non-O139 (AB219453.1, AB219451.1), $V$. cholerae O139 (AB219237.1), P. mirabilis (JX089581.1). The $2.8 \mathrm{~kb}$ variable region of integron was $99 \%$ identical (66\% query coverage) to a sequence from $P$. mirabilis (KF501390.1,$\underline{\text { HQ888851.1 }}$, EU259884.2). Electroporation experiments revealed two types of transformants (class 1 integron-positive and class 1 integron-negative) from the $\mathrm{Pv}$ NBA2365 while one kind of transformant (class 1 integronnegative) was obtained from Vc IDH02365. Antibiogram analysis revealed common resistance profiles of ampicillin, kanamycin, nalidixic acid, neomycin in Vc IDH02365 transformant and integron-negative transformant of Pv NBA2365. Though it still remains to be proved that these organisms shared the same plasmid by virtue of the drug resistance phenotype they conferred upon their respective transformants, there is a possibility that this plasmid was shared between them through horizontal gene transfer.

Therefore, the two organisms utilized all possible mobile genetic elements (SXT element, integrons, and plasmids) to cope with the antibiotic pressure. Though the possibility of other genetic factors in determining antibiotic resistance traits could not be ruled out, a lot more research needs to be carried out to define the complete arsenal that these organisms possessed. In the present study, though Pv NBA2365 did not show pathogenicity as assessed by its FA potential in a rabbit ileal loop assay, it could nevertheless act as a reservoir of multidrug resistance genes. A very recent report has shown the presence of a small plasmid pVERM in P. vermicola and hypothesized that this plasmid could have harbored $q n r D$ allele for quinolone resistance and transferred it to other bacteria during evolution (Guillard et al., 2014). Hence, this is the first report of a highly drug resistant $P$. vermicola $\mathrm{Pv}$ NBA2365 where various genetic elements governing the drug resistance profile of this organism have been unraveled.

\section{AUTHOR CONTRIBUTIONS}

Conceived and designed the experiments: Ashima K. Bhardwaj and Hemanta Koley. Performed the experiments: Neha Rajpara, Braj M. R. N. S. Kutar, Dhrubajyoti Nag, Ritam Sinha, Hemanta Koley. Analyzed the data: Ashima K. Bhardwaj, Thandavarayan Ramamurthy, Hemanta Koley, Neha Rajpara, Braj M. R. N. S. Kutar. Contributed reagents/materials/analysis tools: Ashima K. Bhardwaj, Thandavarayan Ramamurthy, Hemanta Koley. Wrote the paper: Ashima K. Bhardwaj, Thandavarayan Ramamurthy, Braj M. R. N. S. Kutar, Neha Rajpara.

\section{ACKNOWLEDGMENTS}

This work was supported by grant from the Department of Biotechnology (DBT), Ministry of Science and Technology, Government of India [Grant number BT/PR/11634/INF/22/104/2008]. The authors thankfully acknowledge the partial funding received from The Puri Foundation for Education in India. Braj M. R. N. S. Kutar is a recipient of Senior Research Fellowship [Fellowship number 09/988(0003)/2010-EMR-I] from the Council of Scientific and Industrial Research, India. Neha Rajpara is a recipient of Senior Research Fellowship [Grant number AMR/49/11-ECDI] from Indian Council of Medical Research, New Delhi, India. The funders had no role in the study design, in the collection, analysis and interpretation of data; in the writing of the manuscript; and in the decision to submit the manuscript for publication. Support from Prof. Vijay Kumar Chaudhary at DNA Sequencing Facility, University of Delhi South Campus, New Delhi, India, is gratefully acknowledged. Authors also thank Mr. Hardik Upadhyay, Mr. Priyabrata Mohanty, and Mr. Kittappa VinothKumar for their technical support.

\section{REFERENCES}

Akopyanz, N., Bukanov, N. O., Westblom, T. U., Kresovich, S., and Berg, D. E. (1992). DNA diversity among clinical isolates of Helicobacter pylori detected by PCR-based RAPD fingerprinting. Nucleic Acids Res. 20, 5137-5142. doi: $10.1093 /$ nar/20.19.5137

Atlas, R. M. (2010). Handbook of Microbiological Media, 4th Edn. (Boca Raton, FL: CRC Press Inc.).

Beaber, J. W., Burrus, V., Hochhut, B., and Waldor, M. K. (2002). Comparison of SXT and R391, two conjugative integrating elements: definition of a genetic backbone for the mobilization of resistance determinants. Cell. Mol. Life Sci. 59, 2065-2070. doi: 10.1007/s000180200006

Bhardwaj, A. K., and Mohanty, P. (2012). Bacterial efflux pumps involved in multidrug resistance and their inhibitors: rejuvinating the antimicrobial chemotherapy. Recent Pat. Antiinfect. Drug Discov. 7, 73-89. doi: $10.2174 / 157489112799829710$

Bhardwaj, A. K., and Vinothkumar, K. (2015). "Evolution of MDRs," in Quorum Sensing vs Quorum Quenching: A Battle with No End in Sight, ed. V. C. Kalia (New Delhi: Springer), 9-22. doi: 10.1007/978-81-322-1982-8_2

Bhardwaj, A. K., Vinothkumar, K., and Rajpara, N. (2013). Bacterial quorum sensing inhibitors: attractive alternatives for control of infectious pathogens showing multiple drug resistance. Recent Pat. Antiinfect. Drug Discov. 8, 68-83. doi: 10.2174/1574891X11308010012

Bhardwaj, A. K., Vinothkumar, K., Rajpara, N., Mohanty, P., and Kutar, B. M. R. N. S. (2014). "Therapeutic limitations due to antibiotic drug resistance: road to alternate therapies," in Frontiers in Anti-Infective Drug Discovery, Vol. 3, eds Attaur-Rahman and M. I. Choudhary (Sharjah: Bentham Science Publiser), 72-141.

Burrus, V., Marrero, J., and Waldor, M. K. (2006). The current ICE age: biology and evolution of SXT-related integrating conjugative elements. Plasmid 55, 173-183. doi: 10.1016/j.plasmid.2006.01.001

Burrus, V., and Waldor, M. K. (2004). Shaping bacterial genomes with integrative and conjugative elements. Res. Microbiol. 155, 376-386. doi: 10.1016/j.resmic.2004.01.012

Chakraborty, S., Garg, P., Ramamurthy, T., Thungapathra, M., Gautam, J. K., Kumar, C., et al. (2001). Comparison of antibiogram, virulence genes, ribotypes and DNA fingerprints of Vibrio cholerae of matching serogroups isolated from hospitalised diarrhoea cases and from the environment during 1997-1998 in Calcutta, India. J. Med. Microbiol. 50, 879-888.

CLSI. (2010). Performance Standards for Antimicrobial Susceptibility Testing. Wayne, PA: Twentieth informational supplement, M100-S120.

Coetzee, J. N., Datta, N., and Hedges, R. W. (1972). R factors from Proteus rettgeri. J. Gen. Microbiol. 72, 543-552. doi: 10.1099/00221287-72-3-543

Dalsgaard, A., Forslund, A., Petersen, A., Brown, D. J., Dias, F., Monteiro, S., et al. (2000). Class 1 integron-borne, multiple-antibiotic resistance encoded by a 150 kilobase conjugative plasmid in epidemic Vibrio cholerae $\mathrm{O} 1$ strains isolated in Guinea-Bissau. J. Clin. Microbiol. 38, 3774-3779. 
De, S. H., and Chatterje, D. N. (1953). An experimental study of the mechanism of action of Vibrio cholerae on the intestinal mucous membrane. J. Pathol. Bacteriol. 46, 559-562. doi: 10.1002/path.1700660228

Eden, P. A., Schmidt, T. M., Blakemore, R. P., and Pace, N. R. (1991). Phylogenetic analysis of Aquaspirillum magnetotacticum using polymerase chain reaction-amplified 16S rRNA-specific DNA. Int. J. syst. bacterial. 41, 324-325. doi: 10.1099/00207713-41-2-324

Enzensberger, R., Besier, S., Baumgartner, N., and Brade, V. (2005). Mixed diarrhoeal infection caused by Vibrio cholerae and several other enteric pathogens in a 4-yearold child returning to Germany from Pakistan. Scand. J. Infect. Dis. 37, 73-75. doi: 10.1080/00365540510026409

Faruque, A. S., Mahalanabis, D., Islam, A., and Hoque, S. S. (1994). Severity of cholera during concurrent infections with other enteric pathogens. J. Diarrhoeal Dis. Res. 12, 214-218.

Faruque, S. M., Albert, M. J., and Mekalanos, J. J. (1998). Epidemiology, genetics, and ecology of toxigenic Vibrio cholerae. Microbiol. Mol. Biol. Rev. 62, 1301-1314.

Faruque, S. M., Kamruzzaman, M., Meraj, I. M., Chowdhury, N., Nair, G. B., Sack, R. B., et al. (2003). Pathogenic potential of environmental Vibrio cholerae strains carrying genetic variants of the toxin-coregulated pilus pathogenicity island. Infect. Immun. 71, 1020-1025. doi: 10.1128/IAI.71.2.1020-1025. 2003

Ghosh, A., and Ramamurthy, T. (2011). Antimicrobials and cholera: are we stranded? Indian J. Med. Res. 133, 225-231.

Guillard, T., Grillon, A., De Champs, C., Cartier, C., Madoux, J., Bercot, B., et al. (2014). Mobile insertion cassette elements found in small non-transmissible plasmids in proteeae may explain qnrD mobilization. PLoS ONE 9:e87801. doi: 10.1371/journal.pone.0087801

Heidelberg, J. F., Eisen, J. A., Nelson, W. C., Clayton, R. A., Gwinn, M. L., Dodson, R. J., etal. (2000). DNA sequence of both chromosomes of the cholera pathogen Vibrio cholerae. Nature 406, 477-483. doi: 10.1038/350 20000

Hochhut, B., Beaber, J. W., Woodgate, R., and Waldor, M. K. (2001). Formation of chromosomal tandem arrays of the SXT element and R391, two conjugative chromosomally integrating elements that share an attachment site. J. Bacteriol. 183, 1124-1132. doi: 10.1128/JB.183.4.1124-1132.2001

Kaper, J. B., Morris, J. G. Jr., and Levine, M. M. (1995). Cholera. Clin. Microbiol. Rev. 8, 48-86.

Kennedy, J. R., and Richardson, S. H. (1969). Fine structure of Vibrio cholerae during toxin production. J. Bacteriol. 100, 1393-1401.

Koreishi, A. F., Schechter, B. A., and Karp, C. L. (2006). Ocular infections caused by Providencia rettgeri. Ophthalmology 113, 1463-1466. doi: 10.1016/j.ophtha.2006.03.047

Krake, P. R., and Tandon, N. (2004). Infective endocarditis due to Providenca stuartii. South. Med. J. 97, 1022-1023. doi: 10.1097/01.SMJ.0000141308.19 657.BA

Kutar, B. M., Rajpara, N., Upadhyay, H., Ramamurthy, T., and Bhardwaj, A. K. (2013). Clinical isolates of Vibrio cholerae O1 El Tor Ogawa of 2009 from Kolkata, India: preponderance of SXT element and presence of Haitian ctxB variant. PLoS ONE 8:e56477. doi: 10.1371/journal.pone.0056477

Lee, H. H., Molla, M. N., Cantor, C. R., and Collins, J. J. (2010). Bacterial charity work leads to population-wide resistance. Nature 467, 82-85. doi: 10.1038/nature 09354

Lennox, E. S. (1955). Transduction of linked genetic characters of the host by bacteriophage P1. Virology 1, 190-206. doi: 10.1016/0042-6822(55)90016-7

MacFaddin, J. F. (2000). Biochemical Tests for Identification of Medical Bacteria, 3rd Edn. Baltimore, MD: Lippincott Williams \& Wilkins.

Macleod, S. M., and Stickler, D. J. (2007). Species interactions in mixed-community crystalline biofilms on urinary catheters. J. Med. Microbiol. 56, 1549-1557. doi: 10.1099/jmm.0.47395-0

Maguire, A. J., Brown, D. F., Gray, J. J., and Desselberger, U. (2001). Rapid screening technique for class 1 integrons in Enterobacteriaceae and nonfermenting gram-negative bacteria and its use in molecular epidemiology. Antimicrob. Agents Chemother. 45, 1022-1029. doi: 10.1128/AAC.45.4.1022-1029. 2001

Marchandin, H., Carriere, C., Sirot, D., Pierre, H. J., and Darbas, H. (1999). TEM-24 produced by four different species of Enterobacteriaceae, including Providencia rettgeri, in a single patient. Antimicrob. Agents Chemother. 43, 2069-2073.
Mohanty, P., Patel, A., and Bhardwaj, A. K. (2012). Role of H- and DMATE-type transporters from multidrug resistant clinical isolates of Vibrio fluvialis in conferring fluoroquinolone resistance. PLoS ONE 7:e35752. doi: 10.1371/journal.pone.0035752

Morris, G. K., Merson, M. H., Huq, I., Kibrya, A. K., and Black, R. (1979). Comparison of four plating media for isolating Vibrio cholerae. J. Clin. Microbiol. 9, 79-83.

Mukherjee, A. K., Chowdhury, P., Bhattacharya, M. K., Ghosh, M., Rajendran, K., and Ganguly, S. (2009). Hospital-based surveillance of enteric parasites in Kolkata. BMC Res. Notes 2:110. doi: 10.1186/1756-05002-110

Murata, T., Iida, T., Shiomi, Y., Tagomori, K., Akeda, Y., Yanagihara, I., etal. (2001). A large outbreak of foodborne infection attributed to Providencia alcalifaciens. J. Infect. Dis. 184, 1050-1055. doi: 10.1086/ 323458

Nandi, B., Nandy, R. K., Mukhopadhyay, S., Nair, G. B., Shimada, T., and Ghose, A. C. (2000). Rapid method for species-specific identification of Vibrio cholerae using primers targeted to the gene of outer membrane protein OmpW. J. Clin. Microbiol. 38, 4145-4151.

Navaneethan, U., and Giannella, R. A. (2008). Mechanisms of infectious diarrhea. Nat. Clin. Pract. Gastroenterol. Hepatol. 5, 637-647. doi: 10.1038/ncpgasthe p1264

Parsons, M. B., Cooper, K. L., Kubota, K. A., Puhr, N., Simington, S., Calimlim, P. S., et al. (2007). PulseNet USA standardized pulsed-field gel electrophoresis protocol for subtyping of Vibrio parahaemolyticus. Foodborne Pathog. Dis. 4, 285-292. doi: 10.1089/fpd.2007.0089

Rajpara, N., Patel, A., Tiwari, N., Bahuguna, J., Antony, A., Choudhury, I., et al. (2009). Mechanism of drug resistance in a clinical isolate of Vibrio fluvialis: involvement of multiple plasmids and integrons. Int. J. Antimicrob. Agents 34, 220-225. doi: 10.1016/j.ijantimicag.2009.03.020

Rajpara, N., Vinothkumar, K., Mohanty, P., Singh, A. K., Singh, R., Sinha, R., et al. (2013). Synergistic effect of various virulence factors leading to high toxicity of environmental $V$. cholerae non-O1/non-O139 isolates lacking ctx gene: comparative study with clinical strains. PLOS ONE 8:e76200. doi: 10.1371/journal.pone.0076200

Sack, D. A., Lyke, C., McLaughlin, C., and Suwanvanichkij, V. (2001). Antimicrobial Resistance in Shigellosis, Cholera and Campylobacteriosis. Report No: WHO/CDS/CSR/DRS/2001/8. World Health Organization, Geneva.

Singh, R., Rajpara, N., Tak, J., Patel, A., Mohanty, P., Vinothkumar, K., et al. (2012). Clinical isolates of Vibrio fluvialis from Kolkata, India, obtained during 2006: plasmids, the qnr gene and a mutation in gyrase A as mechanisms of multidrug resistance. J. Med. Microbiol. 61, 369-374. doi: 10.1099/jmm.0. 037226-0

Somvanshi, V. S., Lang, E., Straubler, B., Sproer, C., Schumann, P., Ganguly, S., et al. (2006). Providencia vermicola sp. nov., isolated from infective juveniles of the entomopathogenic nematode Steinernema thermophilum. Int. J. Syst. Evol. Microbiol. 56, 629-633. doi: 10.1099/ijs.0.63973-0

Stokes, H. W., and Hall, R. M. (1989). A novel family of potentially mobile DNA elements encoding site-specific gene-integration functions: integrons. Mol. Microbiol. 3, 1669-1683. doi: 10.1111/j.1365-2958.1989. tb00153.x

Thungapathra, M., Amita, Sinha, K. K., Chaudhuri, S. R., Garg, P., Ramamurthy, T., et al. (2002). Occurrence of antibiotic resistance gene cassettes aac(6')-Ib, dfrA5, dfrA12, and ereA2 in class I integrons in non-O1, non-O139 Vibrio cholerae strains in India. Antimicrob. Agents Chemother. 46, 2948-2955. doi: 10.1128/AAC.46.9.2948-2955.2002

Waldor, M. K., Tschape, H., and Mekalanos, J. J. (1996). A new type of conjugative transposon encodes resistance to sulfamethoxazole, trimethoprim, and streptomycin in Vibrio cholerae O139. J. Bacteriol. 178, 4157-4165.

WHO. (1987). Manual of Laboratory Investigation of Acute Enteric Infections. Publication no. WHO/CDD/83.3 rev 1. World Health Organization, Geneva.

WHO. (2014). Antimicrobial Resistance: Global Report on Surveillance 2014. Available at: http://www.who.int/drugresistance/documents/surveillancereport/en/

Yanisch-Perron, C., Vieira, J., and Messing, J. (1985). Improved M13 phage cloning vectors and host strains: nucleotide sequences of the M13mp18 and pUC19 vectors. Gene 33, 103-119. doi: 10.1016/0378-1119(85) 90120-9 
Yoh, M., Matsuyama, J., Ohnishi, M., Takagi, K., Miyagi, H., Mori, K., et al. (2005). Importance of Providencia species as a major cause of travellers' diarrhea. J. Med. Microbiol. 54, 1077-1082. doi: 10.1099/jmm.0.45846-0

Young-Keun, Y., Park, H. W., Shrestha, S., Seo, J., Kim, Y. O., Shin, C. S., et al. (2007). Identification of two entomopathogenic bacteria from a nematode pathogenic to the Oriental beetle, Blitopertha orientalis. J. Microbiol. Biotech. 17, 968-978.

Conflict of Interest Statement: The authors declare that the research was conducted in the absence of any commercial or financial relationships that could be construed as a potential conflict of interest.

Received: 13 August 2014; accepted: 16 January 2015; published online: 17 February 2015
Citation: Rajpara N, Kutar BMRNS, Sinha R, Nag D, Koley H, Ramamurthy T and Bhardwaj AK (2015) Role of integrons, plasmids and SXT elements in multidrug resistance of Vibrio cholerae and Providencia vermicola obtained from a clinical isolate of diarrhea. Front. Microbiol. 6:57. doi: 10.3389/fmicb.2015.00057

This article was submitted to Antimicrobials, Resistance and Chemotherapy, a section of the journal Frontiers in Microbiology.

Copyright (C) 2015 Rajpara, Kutar, Sinha, Nag, Koley, Ramamurthy and Bhardwaj. This is an open-access article distributed under the terms of the Creative Commons Attribution License (CC BY). The use, distribution or reproduction in other forums is permitted, provided the original author(s) or licensor are credited and that the original publication in this journal is cited, in accordance with accepted academic practice. No use, distribution or reproduction is permitted which does not comply with these terms. 